\title{
On the Propagation of Slip Fronts at Frictional Interfaces
}

\section{Journal Article}

Author(s):

Kammer, David S. (D); Yastrebov, Vladislav A.; Spijker, Peter; Molinari, Jean-François

Publication date:

2012-10

Permanent link:

https://doi.org/10.3929/ethz-b-000348419

Rights / license:

In Copyright - Non-Commercial Use Permitted

Originally published in:

Tribology Letters 48(1), https://doi.org/10.1007/s11249-012-9920-0 


\title{
On the Propagation of Slip Fronts at Frictional Interfaces
}

\author{
David S. Kammer • Vladislav A. Yastrebov • \\ Peter Spijker · Jean-François Molinari
}

Received: 14 December 2011/ Accepted: 24 January 2012/Published online: 8 February 2012

(C) Springer Science+Business Media, LLC 2012

\begin{abstract}
The dynamic initiation of sliding at planar interfaces between deformable and rigid solids is studied with particular focus on the speed of the slip front. Recent experimental results showed a close relation between this speed and the local ratio of shear to normal stress measured before slip occurs (static stress ratio). Using a two-dimensional finite element model, we demonstrate, however, that fronts propagating in different directions do not have the same dynamics under similar stress conditions. A lack of correlation is also observed between accelerating and decelerating slip fronts. These effects cannot be entirely associated with static local stresses but call for a dynamic description. Considering a dynamic stress ratio (measured in front of the slip tip) instead of a static one reduces the abovementioned inconsistencies. However, the effects of the direction and acceleration are still present. To overcome this, we propose an energetic criterion that uniquely associates, independently on the direction of propagation and its acceleration, the slip front velocity with the relative rise of the energy density at the slip tip.
\end{abstract}

Keywords Dynamic modelling - Friction mechanisms · Contact mechanics $\cdot$ Stick-slip $\cdot$ Stress analysis

\section{Introduction}

Many aspects in engineering, technology, and science concerning friction have impact on our daily lives [1]. As

\footnotetext{
D. S. Kammer · V. A. Yastrebov $(\bowtie) \cdot$ P. Spijker ·

J.-F. Molinari

Computational Solid Mechanics Laboratory, Ecole

Polytechnique Fédérale de Lausanne, EPFL, LSMS, IIC-ENAC,

IMX-STI, Station 18, 1015 Lausanne, Switzerland

e-mail: vladislav.yastrebov@epfl.ch
}

such frictional motion has been studied for centuries, but a complete physical understanding of friction is still lacking. For instance, the transition from stick to slip (the onset of dynamic sliding) is not well understood. Nevertheless, the initiation of dynamic sliding is an important aspect in many areas of science including fracture mechanics $[2,3]$ and seismology [4-6].

The onset of dynamic sliding is often globally perceived as a uniform transition from sticking to sliding. In reality, however, it is a much more complex phenomenon. The shear stress distribution at an interface is generally nonuniform and reaches therefore the shear strength only at a narrow zone from which it might cause interface rupture. The repetition of such local slip events results in global sliding and provides a possible explanation of stick-slip behavior that is consistent with recent experiments, which showed that global sliding is preceded by local slip propagating over parts of the contact interface [7, 8]. As shown in [8], these repeating precursors increase continuously their zone of propagation until a last precursor breaks the entire interface and causes global sliding. The propagation speed of interface ruptures was observed to range from slow [7-9] to supersonic [3]. Moreover, the front speed of a single slip event can change along the propagation path [7]. By studying the stress field close to the interface, BenDavid et al. [10] observed experimentally that the rupture velocity of the detachment front is coupled to the local ratio of shear stress $\tau_{\mathrm{s}}$ to normal stress $\sigma_{\mathrm{s}}$ measured before slip initiation.

Recently, numerical investigations [11-15] reproduced the general features of the experimental results of $[8,10]$ using simple spring-block models. In this article, we study numerically the initial slip event using a finite element (FE) method (see also [16]), allowing us to access detailed information on the onset of dynamical sliding and to 
re-examine the hypothesis of Ben-David et al. [10] on the correlation between the slip front speed and $\tau_{\mathrm{s}} / \sigma_{\mathrm{s}}$. The advantage of the FE method over the above-mentioned discrete techniques is the ability to reproduce correctly the mechanical behavior of continua (e.g., isotropy, elasticity).

\section{Model Set-Up}

The two-dimensional system under consideration consists of a rectangular isotropic elastic plate $(w=200 \mathrm{~mm}, h=100$ $\mathrm{mm}$ ) in contact with a rigid plane (see Fig. 1a). The corners of the plate are rounded to avoid stress singularities at the edges. To study this system, we use a FE method with an explicit Newmark- $\beta$ integration scheme [17] in plane stress incorporating an energy conserving contact algorithm. The material properties (see Table 1) correspond to polymethylmethacrylate (PMMA) glass which was also used in the experiments [10]. We employ Rayleigh damping [18, 19] with mass and stiffness proportionality coefficients of 0 and $0.1 \mu \mathrm{s}$, respectively. The deformable solid is discretized by regular quadrilateral elements (with element side ranging for different meshes from 0.67 to $2 \mathrm{~mm}$ ) interpolating the displacement field linearly.

A linearly distributed vertical displacement $\left(u_{y}^{1}=\right.$ $0.37 \mathrm{~mm}, u_{y}^{2}=0.037 \mathrm{~mm}$ ) is imposed at the top of the plate (see Fig. 1a). This loading is, after reaching equilibrium, complemented by applying a uniform horizontal velocity $v_{x}=10^{-6} c_{\mathrm{L}}$, where $c_{\mathrm{L}}$ is the longitudinal wave speed in the deformable solid. The small value of the applied velocity insures quasi-static loading conditions, similar to the experiments [10]. The resulting stress distribution at the interface is nonuniform. Figure $1 \mathrm{~b}$ is a schematic depiction of the ratio of the local tangential traction $t_{\mathrm{s}}$ to the contact
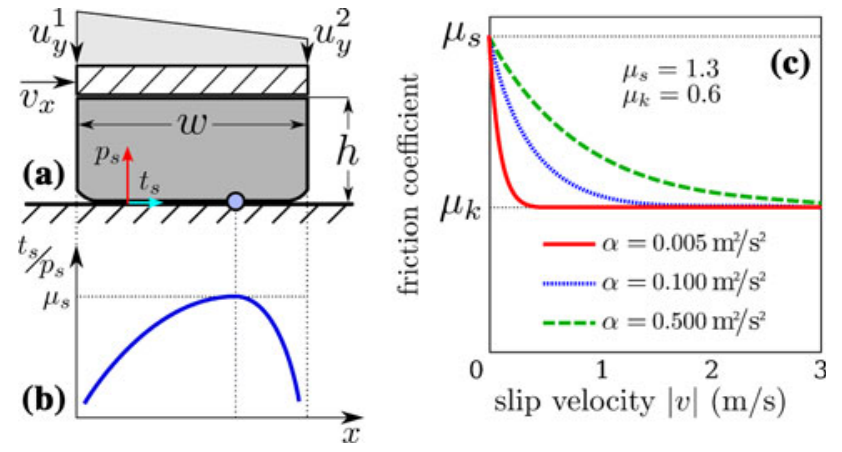

Fig. 1 Two-dimensional set-up of the problem: a a thin rectangular plate in contact with a rigid plane is loaded on the top by a linearly distributed imposed displacement $u_{y}$ and a uniform velocity $v_{x}$; $\mathbf{b}$ the nonuniform distribution of shear to normal tractions $\left(t_{\mathrm{s}}\right.$ and $p_{\mathrm{s}}$, respectively) at the interface causes a first slip nucleation far from the edges $\left[t_{\mathrm{s}} / p_{\mathrm{s}}>\mu_{\mathrm{s}}\right.$ marked by a circle in (a)]; $\mathbf{c}$ the change of the friction coefficient with respect to the material slip velocity $v$ is governed by the parameter $\alpha$ (see Eq. 1)
Table 1 Friction and material parameters corresponding to PMMA glass

\begin{tabular}{ll}
\hline Parameter & \\
\hline Material & \\
Young's modulus $E$ & $2.6 \mathrm{GPa}$ \\
Poisson's ratio $v$ & 0.37 \\
Density $\rho$ & $1200 \mathrm{~kg} / \mathrm{m}^{3}$ \\
Longitudinal wave speed $c_{\mathrm{L}}$ & $1584 \mathrm{~m} / \mathrm{s}$ \\
Transverse wave speed $c_{\mathrm{S}}$ & $890 \mathrm{~m} / \mathrm{s}$ \\
Friction & \\
Static friction coefficient $\mu_{\mathrm{s}}$ & 1.3 \\
Kinetic friction coefficient $\mu_{\mathrm{k}}$ & $0.6 ; 1.0$ \\
Transition parameter $\alpha$ & $0.1 \mathrm{~m}^{2} / \mathrm{s}^{2}$ \\
\hline
\end{tabular}

pressure $p_{\mathrm{s}}$. These tractions (denoted with a subscript $\mathrm{s}$ ) are measured at the moment preceding interface rupture and are referred to hereafter as static. The imposed loading conditions insure a spontaneous nucleation of the first slip event inside the contact interface far from the edges (circle in Fig. 1a), because this is where the nonsymmetric stress distribution reaches a critical value $t_{\mathrm{s}} / p_{\mathrm{s}}>\mu_{\mathrm{s}}$, see Fig. $1 \mathrm{~b}$. In the stick state, the tangential resistance of the interface is assumed to be proportional to the contact pressure $p$ with a coefficient $\mu_{\mathrm{s}}$. As for the slip state, this coefficient of proportionality $\mu$ is determined by the velocity ( $v$ ) weakening friction law (see Fig. 1c)

$\mu=\mu_{\mathrm{k}}+\left(\mu_{\mathrm{s}}-\mu_{\mathrm{k}}\right) \exp \left(-|v| \sqrt{\left(\mu_{\mathrm{s}}-\mu_{\mathrm{k}}\right) / \alpha}\right)$,

which insures a smooth transition from the static $\mu_{\mathrm{s}}$ to the kinetic $\mu_{\mathrm{k}}$ friction coefficient governed by the transition parameter $\alpha$. The parameters of the friction law are as well presented in Table 1. The local $\mu_{\mathrm{s}}$ corresponds to experimental results as reported in Fig. 4a, b in [20] and is considerably higher than the global static coefficient of friction; an effect that was also observed in spring-block simulations $[12,21]$. The local kinetic friction coefficient as well as the transition parameter were not measured in the experiments. Therefore, they were studied here qualitatively (see first paragraph of the following section) and eventually chosen arbitrarily. When the ratio of the local tangential traction to the contact pressure exceeds the static friction threshold $\left(t_{\mathrm{s}} / p_{\mathrm{s}}>\mu_{\mathrm{s}}\right)$, slip occurs and propagates in one or both directions along the frictional interface. The dynamics of the slip fronts are determined by the parameters of the friction law (Eq. 1) as well as by the local stress state.

\section{Results and Discussion}

We have conducted several simulations (not all presented in this article) and have observed different types of slip: 
crack-like (the entire interface between the crack tips is slipping), pulse-like (the slip region propagates along the interface within a narrow pulse), and mixed modes when a crack converts to pulses and vice versa. The propagation speed of the slip tip $V_{\text {tip }}$ is related to the local stress state and seems not to depend on the type of slip. By studying the influence of the friction law parameters, we have observed that for an increasing (decreasing) difference between the static and the kinetic friction coefficients $\Delta \mu=$ $\mu_{\mathrm{s}}-\mu_{\mathrm{k}}$, the slip type tends to be crack-like (pulse-like). A higher transition parameter $\alpha$ causes slower slip propagation especially during slip initiation and slip arrest.

In order to compare our numerical results with the experimental observations of Ben-David et al. [10], we present $V_{\text {tip }}$ as a function of the ratio of shear to normal stress measured before slip initiation. Here, the slip tip speed $V_{\text {tip }}$ is normalized to the longitudinal wave speed $c_{\mathrm{L}}$ and the local stress ratio is replaced by the local static ratio of tangential surface traction $t_{\mathrm{s}}$ to contact pressure $p_{\mathrm{s}}$ (see Fig. 2). Our results confirm the experimentally [10] and numerically [14] observed general trend that the rupture propagation is faster for higher $t_{\mathrm{s}} / p_{\mathrm{s}}$ ratios. For friction parameters $\mu_{\mathrm{s}}=1.3, \mu_{\mathrm{k}}=1.0$, and $\alpha=0.1 \mathrm{~m}^{2} / \mathrm{s}^{2}$ the slip front velocities are in good quantitative agreement with the experimental results. Consistently with experiments [10], for the given type of loading (only at the top face), we do not observe slow fronts. Interestingly, we note that the rupture propagates considerably slower in the direction of the imposed shear load than in the opposite direction (compare solid with dashed line in Fig. 2). These differences have not been reported in the experiments.

To enable the separation of effects due to slip directionality and any other sources that might cause a nonunique relation between the $t_{\mathrm{s}} / p_{\mathrm{s}}$ ratio and the rupture propagation speed, we consider two additional simulations

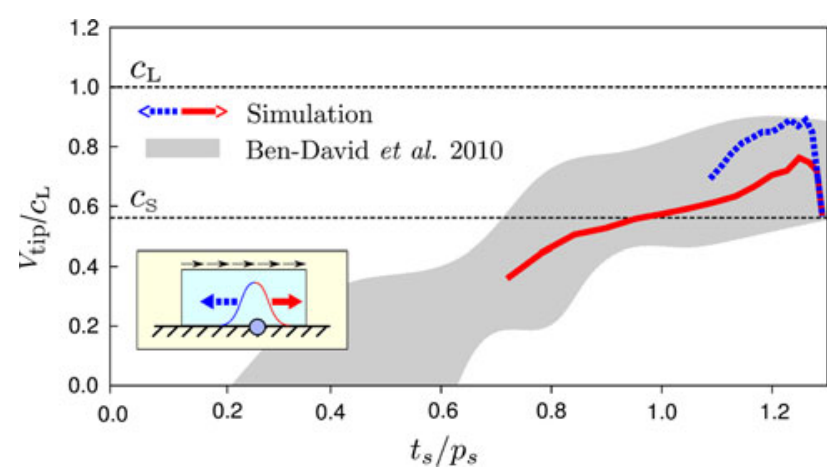

Fig. 2 Comparison of numerical results with experimental observations by Ben-David et al. [10]. The normalized rupture velocity is reported with respect to the static ratio of local tangential surface traction $t_{\mathrm{s}}$ to contact pressure $p_{\mathrm{s}}$. Friction parameters are $\mu_{\mathrm{s}}=1.3, \mu_{\mathrm{k}}=1.0$, and $\alpha=0.1 \mathrm{~m}^{2} / \mathrm{s}^{2}$
(Fig. 3), where slip events are triggered at the edges. In order to increase the propagation distance (in comparison to Fig. 2, where the rupture propagating in the opposite direction of the imposed shear load arrests not far from the initiation zone) the kinetic coefficient of friction is reduced resulting in the following set of friction parameters: $\mu_{\mathrm{s}}=1.3, \mu_{\mathrm{k}}=0.6$, and $\alpha=0.1 \mathrm{~m}^{2} / \mathrm{s}^{2}$. In all three cases, the loading history of the body is identical up to the moment the tangential surface traction reaches the friction threshold, i.e., the initial stress state is the same for all simulations (see solid line in Fig. 4). The slip propagation is then triggered by manually increasing the local tangential surface traction within small nucleation zones at the edges (Fig. 3a, c). Otherwise if the global shear load is slightly increased, rupture nucleates spontaneously far from the edges as before (Fig. 3b). In case of spontaneous initiation (Fig. 3d, solid line), the rupture propagates fast toward the edges and its velocity decreases along the path with a decreasing ratio $t_{\mathrm{s}} / p_{\mathrm{s}}$. Note that under some conditions, we observe supersonic slip fronts, which were not observed in [10]. However, our results are consistent with rupture in bi-material interfaces where the stiffer material limits the propagation speed as observed experimentally and numerically by Coker et al. [22]. For the two edgetriggered ruptures, the slip propagates relatively slowly in the first phase, accelerates, reaches a maximum value (for maximal ratio $t_{\mathrm{s}} / p_{\mathrm{s}}$ ) and decelerates afterward (see Fig. 3d, dashed and dashed-dotted lines). Although the triggered ruptures are unidirectional, there is no unique slip tip speed associated with a given $t_{\mathrm{s}} / p_{\mathrm{s}}$ value. The maximal rupture velocity of the left-triggered slip does not exceed $60 \%$ of the maximal speed for the other two cases.

As seen most clearly in Fig. 3a, the slip front (marked by a small white triangle) propagating at super-shear velocity follows the longitudinal wave (the circular white zone furthest from the nucleation zone), which modifies the local stress state at the interface. Therefore, looking at the dynamic ratio $t_{\mathrm{d}} / p_{\mathrm{d}}$ measured in front of the slip tip, instead of examining the static ratio $t_{\mathrm{s}} / p_{\mathrm{s}}$, would allow to account for the dynamic nature of the slip propagation.

Here, the location of the slip tip is determined to coincide with the position of the sticking node in front of the slipping nodes (see inset in Fig. 4). According to this definition, the position of the rupture tip changes abruptly when the front advances. However, its velocity is computed in a continuous way as $V_{\text {tip }}=l^{*} / \Delta t$, where $l^{*}$ is a characteristic distance (here $l^{*}=0.67 \mathrm{~mm}$ ) and $\Delta t$ is the time interval that the rupture needs to advance this distance.

In the context of discrete contact, we propose to analyze an instantaneous dynamic stress state $\left(t_{\mathrm{d}}\right.$ and $\left.p_{\mathrm{d}}\right)$ at the slip tip right after it jumps to a new position (see inset in 

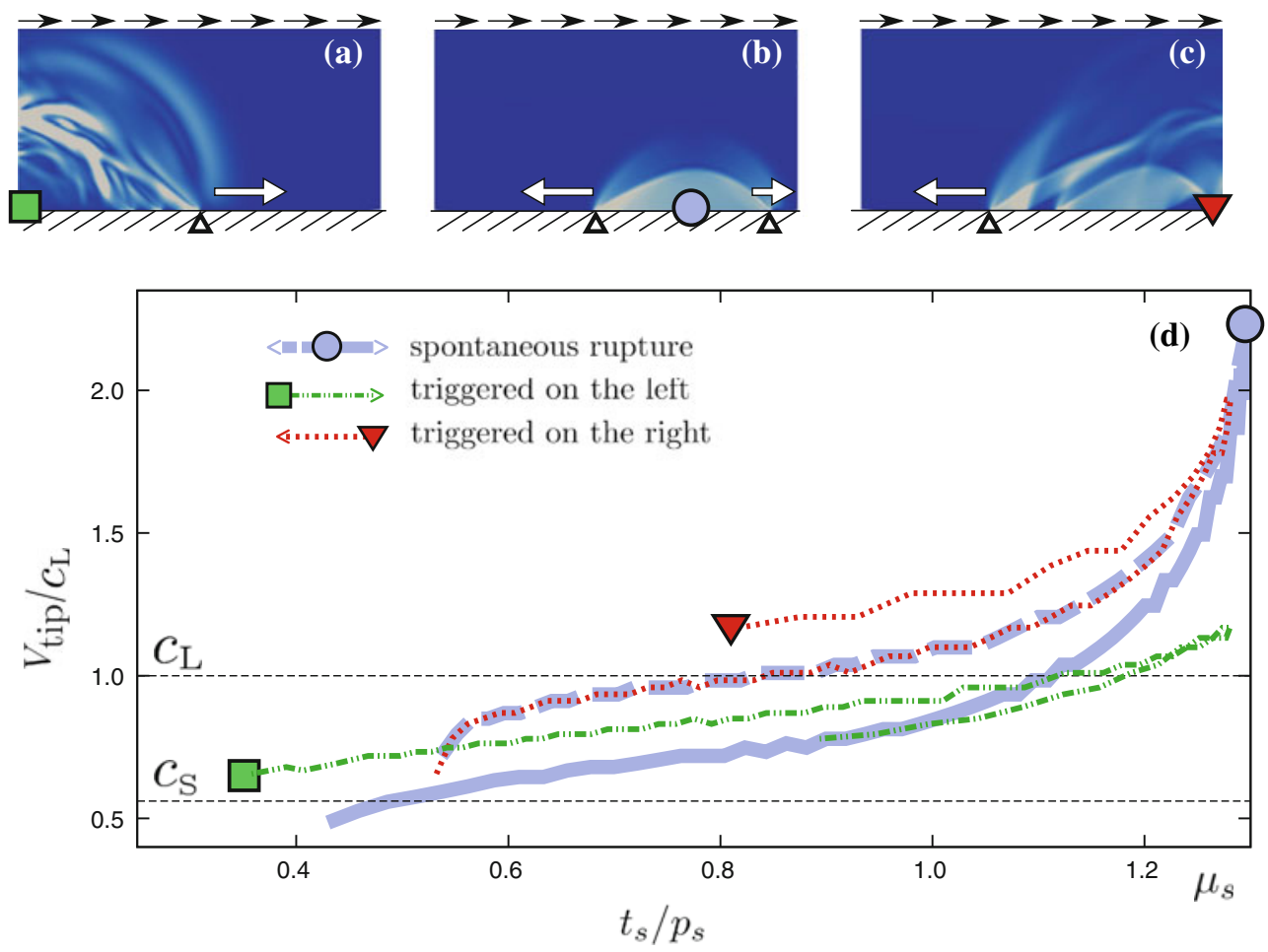

Fig. 3 Three different slip events are presented for the same initial stress state (before triggering or spontaneous initiation). Instantaneous material velocity is shown for the slip event a triggered at the left edge, $\mathbf{b}$ spontaneously initiated far from the edges, and $\mathbf{c}$ triggered at the right edge. Colors from blue to white denote material velocities ranging from 0 to $2 \mathrm{~m} / \mathrm{s}$, respectively. The starting point of each event is marked with a square, a circle, and a triangle, respectively. Small white triangles show the location of the tip of the slip front. Black

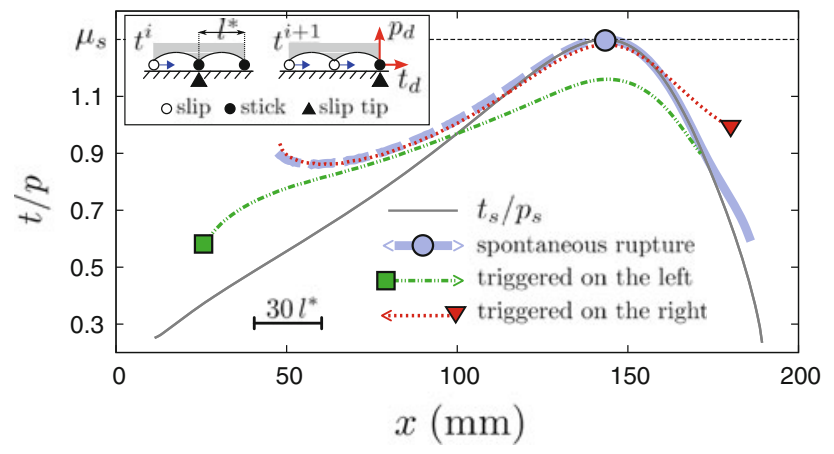

Fig. 4 For each point along the interface $x$, the dynamic stress ratio $t_{\mathrm{d}} / p_{\mathrm{d}}$ is plotted at the moment when the slip front arrives at this location. Note that contrary to the reported static stress ratio $t_{\mathrm{s}} / p_{\mathrm{s}}$, this is not an instantaneous picture but an assembly of results over the entire time of propagation. Data close to the triggering zones are omitted. Inset the dynamic values $t_{\mathrm{d}}$ and $p_{\mathrm{d}}$ are measured at the sticking node in front of the slipping region at the moment $t^{i+1}$ when the previous node starts to slip

Fig. 4). The dynamic ratio $t_{\mathrm{d}} / p_{\mathrm{d}}$ differs significantly from the static one (compared in Fig. 4), being changed by the longitudinal wave often preceding the slip front. It is worth noting that the value of the dynamic ratio is far from the arrows indicate the direction of the imposed global shear load, whereas white arrows show the direction of the rupture propagation. $\mathbf{d}$ The normalized rupture velocity for all three cases is depicted with respect to the local static ratio of tangential traction $t_{\mathrm{s}}$ to contact pressure $p_{\mathrm{s}}$ (data close to the triggering zone are not shown). Friction parameters are $\mu_{\mathrm{s}}=1.3, \mu_{\mathrm{k}}=0.6$, and $\alpha=0.1 \mathrm{~m}^{2} / \mathrm{s}^{2}$ (Color figure online)

critical value $\mu_{\mathrm{s}}$ for a large part of the propagation path, which implies the need for a strong change of the stress state at the rupture tip within a short time.

The relation between the velocity of the slip front and the dynamic ratio $t_{\mathrm{d}} / p_{\mathrm{d}}$ is depicted in Fig. 5. Compared to Fig. 3, the rupture triggered on the left is in better agreement with the other two (faster) slip fronts. Particularly, the slopes are more consistent for all curves and the range of velocities is smaller for a given ratio $t_{\mathrm{d}} / p_{\mathrm{d}}$. Again it is confirmed that the character of the slip propagation is directionality dependent. For a given ratio $t_{\mathrm{d}} / p_{\mathrm{d}}$, the slip fronts propagating in the direction opposite to the sliding are faster than the oncoming fronts (in Fig. 5, e.g., compare the dashed with the dashed-dotted curves). Nonetheless, the difference between the curves cannot be only attributed to the directionality (in Fig. 5, note the two branches of the dashed and dashed-dotted curves). The accelerating slip fronts show a faster rupture velocity than the decelerating ones for the same given ratio $t_{\mathrm{d}} / p_{\mathrm{d}}$. Further, the general trend of faster rupture for higher $t / p$ is lost (enclosed by the large circle in Fig. 5); at a certain moment, the rupture speed starts to decrease rapidly with increasing $t_{\mathrm{d}} / p_{\mathrm{d}}$ along the propagation path. We observe this phenomenon only 


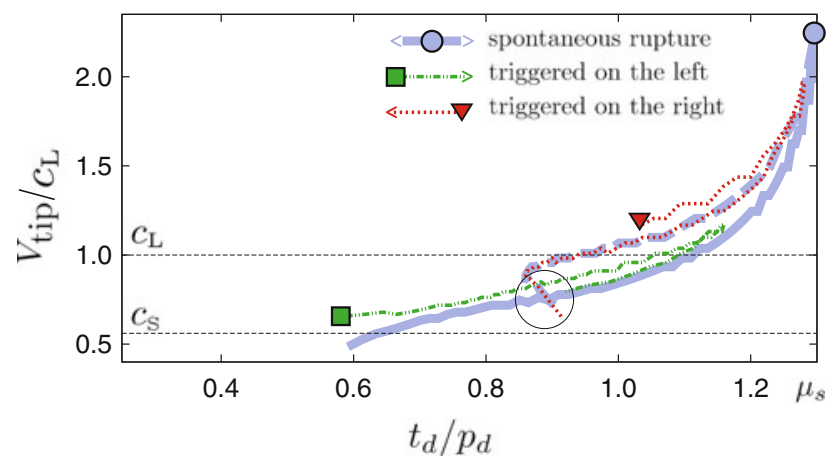

Fig. 5 The normalized rupture velocity is plotted with respect to the dynamic traction ratio $t_{\mathrm{d}} / p_{\mathrm{d}}$ for all three slip events. Data close to the triggering zones are omitted

for slip fronts advancing against the sliding direction. Regardless of the simplicity of the static criterion $t_{\mathrm{s}} / p_{\mathrm{s}}$ and the consistency of the dynamic criterion $t_{\mathrm{d}} / p_{\mathrm{d}}$, a stress ratio does not seem able to provide a fully reliable estimation of the velocity of the slip propagation.

The lack of generality of the velocity criteria based on the ratio of the tangential traction to the contact pressure $t / p$ suggests an independent consideration of $t$ and $p$. It was proposed [10] that the propagation of the slip front is related to the energy densities $U_{\mathrm{s}}$, stored at the front tip, and $U_{\mathrm{r}}$, needed to advance the slip front. We propose a heuristic energy density at the contact interface as

$U(p, t)=\left(2(1+v) t^{2}+p^{2}\right) / 2 E$.

The density of stored energy $U_{\mathrm{s}}=U\left(p_{\mathrm{d}}, t_{\mathrm{d}}\right)$ is measured locally at the slip tip at the moment the front advances one length parameter $l^{*}$, similarly to the dynamic ratio $t_{\mathrm{d}} / p_{\mathrm{d}}$. The density of rupture energy $U_{\mathrm{r}}=U\left(p_{\mathrm{r}}, \mu_{\mathrm{s}} p_{\mathrm{r}}\right)$ is computed at the same material point just before the front advances another $l^{*}$, i.e., when the ratio of tangential traction to contact pressure reaches the static coefficient of friction $\left(t_{\mathrm{r}} / p_{\mathrm{r}}=\mu_{\mathrm{s}}\right)$ (see inset in Fig. 6).

The normalized rupture velocity is depicted in Fig. 6 as a function of the change of the energy density at the slip tip $\Delta U=U_{\mathrm{r}}-U_{\mathrm{s}}$ normalized by the stored energy density $U_{\mathrm{s}}$. The data of all three cases collapse within a narrow region properly described by

$V_{\text {tip }} / c_{L}=a+b \exp \left(-c \sqrt{\Delta U / U_{\mathrm{s}}}\right)$,

where $a, b$, and $c$ are fitting parameters (see Fig. 6). No differences due to the directionality of the slip propagation nor any other reason that caused branching for the previously studied criteria are now present. This shows that the energy density criterion is able to account for the dynamics of slip events at bi-material interfaces. Note that tails of data points falling outside of the fit range occur when the slip fronts start to decelerate rapidly before arresting.

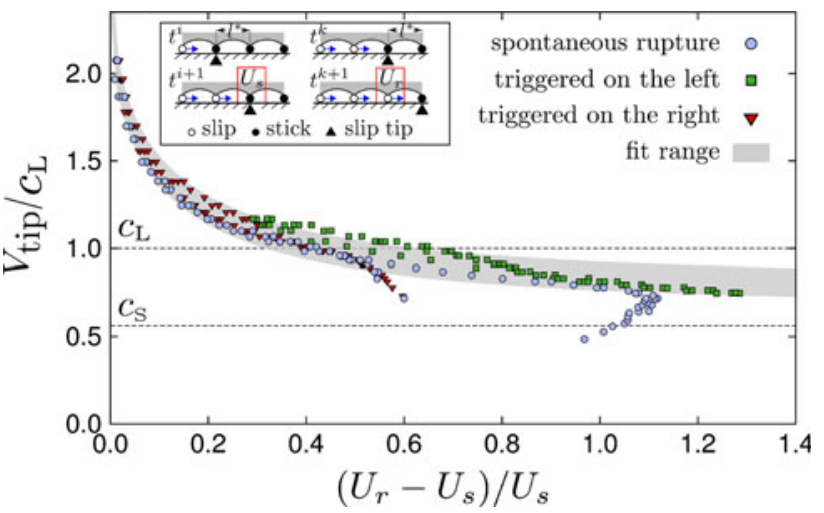

Fig. 6 The normalized rupture velocity is depicted with respect to a dynamic criterion based on a heuristic surface energy density $U$. The ratio $\left(U_{\mathrm{r}}-U_{\mathrm{s}}\right) / U_{\mathrm{s}}$ represents the proportion of the energy change $U_{\mathrm{r}}-U_{\mathrm{s}}$ at the slip tip needed to advance the rupture front with respect to the locally stored energy $U_{\mathrm{s}}$. The gray area is a data fit based on Eq. 3 with $a=0.76 \pm 0.07, b=1.80$, and $c=3.05$. Inset $U_{\mathrm{s}}$ and $U_{\mathrm{r}}$ are measured when the slip tip, respectively, reaches the observation point and overpasses it

\section{Conclusion}

In this article, it is demonstrated that the static ratio of shear to normal stress [10, 14] is not a sufficient criterion for determining the speed of slip fronts. The use of the dynamic ratio, measured in front of the slip tip, improves the estimation of this speed. However, for our set-up we observed that, given a stress ratio (static or dynamic), the front going in the direction of the sliding is always slower than the front propagating in the opposite direction. Moreover, the decelerating fronts are also slower than the accelerating ones. The energetic criterion we propose eliminates these effects and highlights the similarities between the rupture of frictional interfaces [3] and crack propagation [22]. It is hoped that these findings motivate experimental work to access dynamic stress field measurements as well as theoretical studies to extend the principles of fracture mechanics to problems of frictional sliding.

Acknowledgments The authors are grateful to D. Coker for fruitful discussions and N. Richart for helpful advices on the simulations. The research described in this article is supported by the European Research Council (ERCstg UFO-240332).

\section{Reference}

1. Blau, P.J.: Friction Science and Technology, 2nd edn. CRC Press, Boca Raton (2009)

2. Xia, K., Rosakis, A.J., Kanamori, H.: Laboratory earthquakes: the sub-Rayleigh-to-supershear rupture transition. Science 303, 1859-1861 (2004)

3. Coker, D., Lykotrafitis, G., Needleman, A., Rosakis, A.J.: Frictional sliding modes along an interface between identical elastic 
plates subject to shear impact loading. J. Mech. Phys. Solids 53, 884-922 (2005)

4. Heaton, T.H.: Evidence for and implications of self-healing pulses of slip in earthquake rupture. Phys. Earth Planet. Inter. 64, 1-20 (1990)

5. Ben-Zion, Y.: Dynamic ruptures in recent models of earthquake faults. J. Mech. Phys. Solids 49, 2209 (2001)

6. Scholz, C.H.: The Mechanics of Earthquakes and Faulting, 2nd edn. Cambridge University Press, Cambridge (2002)

7. Rubinstein, S.M., Cohen, G., Fineberg, J.: Detachment fronts and the onset of dynamic friction. Nature 430, 1005-1009 (2004)

8. Rubinstein, S., Cohen, G., Fineberg, J.: Dynamics of precursors to frictional sliding. Phys. Rev. Lett. 98, 226103 (2007)

9. Baumberger, T., Caroli, C., Ronsin, O.: Self-healing slip pulses along a gel/glass interface. Phys. Rev. Lett. 88, 075509 (2002)

10. Ben-David, O., Cohen, G., Fineberg, J.: The dynamics of the onset of frictional slip. Science 330, 211 (2010)

11. Braun, O., Barel, I., Urbakh, M.: Dynamics of transition from static to kinetic friction. Phys. Rev. Lett. 103, 194301 (2009)

12. Maegawa, S., Suzuki, A., Nakano, K.: Precursors of global slip in a longitudinal line contact under non-uniform normal loading. Tribol. Lett. 38, 313 (2010)

13. Bouchbinder, E., Brener, E., Barel, I., Urbakh, M.: Slow cracklike dynamics at the onset of frictional sliding. Phys. Rev. Lett. 107, 235501 (2011)
14. Trømborg, J., Scheibert, J., Amundsen, D., Thøgersen, K., Malthe-Sørenssen, A.: Transition from static to kinetic friction: insights from a 2D model. Phys. Rev. Lett. 107, 074301 (2011)

15. Amundsen, D.S., Scheibert, J., Thøgersen, K., Trømborg, J., Malthe-Sørenssen, A.: 1D model of precursors to frictional stickslip motion allowing for robust comparison with experiments. Tribol. Lett. 45, 357 (2012)

16. Di Bartolomeo, M., Meziane, A., Massi, F., Baillet, L., Fregolent, A.: Dynamic rupture at a frictional interface between dissimilar materials with asperities. Tribol. Int. 43, 1620 (2010)

17. Belytschko, T., Liu, W.K., Moran, B.: Nonlinear Finite Elements for Continua and Structures. Wiley, Chichester (2000)

18. Rayleigh, J.W.S.: The Theory of Sound, vol. 1, 2nd edn. Dover Publications, New York (1945)

19. Caughey, T.K.: Classical normal modes in damped linear dynamic systems. J. Appl. Mech. 27, 269-271 (1960)

20. Ben-David, O., Fineberg, J.: Static friction coefficient is not a material constant. Phys. Rev. Lett. 106, 254301 (2011)

21. Scheibert, J., Dysthe, D.K.: Role of friction-induced torque in stick-motion. Europhys. Lett. 92, 54001 (2010)

22. Coker, D., Rosakis, A.J., Needleman, A.: Dynamic crack growth along a polymer composite-Homalite interface. J. Mech. Phys. Solids 51, 425-460 (2003) 